\title{
AVALIAÇÃO DA DOR EM MULHERES COM CÂNCER DE MAMA SUBMETIDAS À EXÉRESE DA REDE LINFÁTICA AXILAR
}

\author{
Vagnára Ribeiro Silva ${ }^{1}$, Clícia Valim Côrtes Gradim²
}

\begin{abstract}
RESUMO: Estudo descritivo-exploratório que teve como objetivo avaliar a intensidade e características do quinto sinal vital, a dor, em 20 mulheres com câncer de mama submetidas à cirurgia mamária com exérese linfática axilar no período de junho a setembro de 2008. Realizado por meio da aplicação de formulário. A dor foi relatada por $50 \%$ das mulheres como presente em algum momento entre o diagnóstico e o tratamento, sendo o período cirúrgico considerado o mais crítico. Em relação à intensidade, predominou pouca dor (40\%). Na qualidade da dor, os descritores sensitivos mais citados foram fisga (16,22\%) e ferroa (16,22\%); nos avaliativos, prevaleceram adormece (20\%) e irradia (14,29\%); e, nos emocionais, predominou incômoda (17,65\%). Conclui-se que a dor é um sintoma presente, que deve ser avaliado pelo enfermeiro como rotina durante o atendimento, com o objetivo de proporcionar intervenção eficaz.
\end{abstract}

PALAVRAS-CHAVE: Neoplasias da mama; Medição da dor; Enfermagem.

\section{EVALUATION OF PAIN IN WOMEN WITH BREAST CANCER SUBMITTED TO RESECTION OF AXILLARY LYMPH NODES}

\begin{abstract}
An exploratory descriptive study aimed to evaluate the intensity and characteristics of the fifth vital sign, pain, in 20 women with breast cancer undergoing breast surgery with axillary lymphatic excision in the period from June to September 2008. It was accomplished through the application form. The pain was reported by $50 \%$ of the women as present at some time between diagnosis and treatment, the surgical period is considered the most critical. Regarding the intensity, low pain predominated (40\%). As for its quality, the sensory descriptors most frequently cited were: slingshot (16.22\%) and stings (16.22\%) among the evaluative descriptors prevailed asleep (20\%) and radiates (14.29\%) and among the emotional ones predominated uncomfortable (17.65\%). It is concluded that pain is a present symptom, which should be evaluated by nurses during routine care with the goal of providing effective intervention.
\end{abstract}

KEYWORDS: Breast neoplasms; Pain measurement; Nursing.

\section{EVALUACIÓN DEL DOLOR EN MUJERES CON CÁNCER DE MAMA SOMETIDAS A EXERESE DE LA RED LINFÁTICA AXILAR}

RESUMEN: Estudio descriptivo-exploratorio que tuvo como objetivo evaluar la intensidad y características del quinto señal vital, el dolor, en 20 mujeres con cáncer de mama sometidas a quirurgia mamaria con exerese linfática axilar en periodo de junio a septiembre de 2008. Realizado por medio de aplicación de formulario. El dolor fue referido por $50 \%$ de las mujeres como presente en algún momento entre el diagnóstico y el tratamiento, siendo el periodo quirúrgico considerado el más crítico. Acerca de la intensidad, ha predominado poco dolor (40\%). En la cualidad del dolor, los descriptores sensitivos más referidos fueron fisgar (16,22\%) y aguijonear (16,22\%); en las evaluaciones, prevalecieron dormirse (20\%) y irradiar (14,29\%); y, en los emocionales, ha predominado incómoda (17,65\%). Se concluye que el dolor es un síntoma presente, que debe ser evaluado por el enfermero como rutina durante el atendimiento con el objetivo de proporcionar intervención eficaz.

PALABRAS CLAVE: Neoplasias de la mama; Contraste del dolor; Enfermería.

${ }^{1}$ Acadêmica do Curso de Graduação em Enfermagem da Universidade Federal de Alfenas-UNIFAL.

${ }^{2}$ Enfermeira. Doutor em Enfermagem. Professor Associado do Departamento de Enfermagem da UNIFAL.

\author{
Autor correspondente: \\ Vagnára Ribeiro da Silva \\ Universidade Federal de Alfenas \\ R. João Florentino da Silva, 87 - 37130-000 - Alfenas-MG-Brasil \\ E-mail: vagnararibeiro@gmail.com
}

Recebido: 09/12/09 Aprovado: 03/08/10 


\section{INTRODUÇÃO}

A dor, conhecida na atualidade como o quinto sinal vital, é de importância e relevância na vida dos pacientes, sendo definida como uma experiência sensorial e emocional desagradável. Ela está relacionada a lesões teciduais, reais ou potenciais, e envolve aspectos psicoafetivos, mecanismos fisiológicos de percepção e causa orgânica específica ${ }^{(1-2)}$.

A dor é uma interpretação do aspecto físicoquímico do estímulo nocivo e da interação deste com as características individuais, enquanto que o sofrimento é a interação da percepção aversiva com as mudanças no funcionamento social, psicológico e cultural, que incluem perda de confiança, medo da morte ou da incapacidade física, de se tornar inválido, isolamento e preocupações financeiras ${ }^{(3)}$.

Atualmente, percebe-se que avaliar a dor do paciente permite uma melhora do atendimento, pois se observa a sua postura e seu comportamento, seus apelos inaudíveis, além de sua verbalização e descrição, tornando-o ativo a ponto de, dentre outros, informar os profissionais de saúde sobre os seus sentimentos ${ }^{(4)}$.

Diferentes métodos têm sido utilizados para a mensuração da dor, sendo utilizadas escalas. As escalas unidirecionais são métodos simplistas por considerar apenas a intensidade da queixa álgica; as multidimensionais enfocam as três dimensões da dor: sensorial-discriminativa, motivacional-afetiva e cognitiva-avaliativa e, por isso, têm boa aceitação de uso nos serviços de saúde ${ }^{(5-6)}$. A escala multidimensional de McGuill, utilizada neste trabalho, apresenta descritores com alto grau de concordância e fidedginidade sobre as relações de dor, sendo utilizada como instrumento experimental para a análise de efeitos de várias condutas de alívio da dor ${ }^{(5-6)}$.

Em oncologia, a dor não é um sintoma característico para os pacientes no início da doença; à medida que a doença vai progredindo e/ou durante seu tratamento, ela passa a ser uma queixa recorrente. É importante enfatizar que muitos pacientes oncológicos não têm dor, mas sofrem de um somatório da dor somática, psicológica, psicossocial e espiritual ${ }^{(2)}$.

A queixa álgica pode ser diretamente relacionada ao tumor, secundária à evolução da patologia, aos procedimentos terapêuticos e diagnósticos e aos aspectos psicoafetivos associados a uma doença que gera uma mudança drástica e repentina na vida do doente e de seus familiares; ela e se constitui em uma síndrome debilitante, progressiva e muitas vezes terminal ${ }^{(7-8)}$.
Em nossa experiência, trabalhando com mulheres com câncer de mama no Núcleo de Ensino, Pesquisa e Extensão Mulher e Câncer de Mama (MUCAMA), do Departamento de Enfermagem da Universidade Federal de Alfenas-MG, percebeu-se que sintomas dolorosos eram queixa comum e causa principal da procura pelo grupo de apoio.

Verificou-se que as mulheres chegavam relatando dor e relacionando-a à má postura corporal, dificuldade de movimentação com o braço homolateral à cirurgia, retirada da rede linfática, ocorrências do linfedema e suas consequências, lesões de algum nervo durante a cirurgia e no local onde ficou o dreno aspirativo.

Essas observações nos levaram a verificar o porquê de tais causas, tendo em vista o câncer de mama ser, em sua maior parte, indolor, em especial nos estágios mais precoces. Comprovou-se, pela literatura, que a mulher, ao ser comunicada de que apresenta um tumor maligno, inconscientemente e como mecanismo de defesa, responde com uma contratura dos músculos da região escapular, o que altera a postura corporal e causa dor nesta região(7).

As complicações cirúrgicas podem remeter à posição da paciente durante o ato cirúrgico, com a extrema amplitude do membro superior. Essa posição contribui para o desenvolvimento de retrações, lesões musculotendinosas e articulares pós-operatórias. Pode ocorrer a colocação errônea de um dreno aspirativo, causando uma lesão ao nervo intercostobraquial e provocar dor devido ao mau posicionamento, exacerbada pelas mudanças de posição, pela mobilização escapular e pelos movimentos inspiratórios ${ }^{(9)}$.

Associado à cirurgia, tem-se o tratamento adjuvante, ambos responsáveis pelo mal-estar, desconforto, medo, entre outros sentimentos que acabam gerando a dor. Nele se incluem as sessões de quimioterapia, que levam à toxicidade sistêmica; as de radioterapia, que podem ocasionar as radiodermites ou radioepitelites; e a hormonioterapia, em que o protocolo de tratamento dura, geralmente, cinco anos, o que acaba causando ansiedade das pacientes, que podem vir a apresentar queixas dolorosas ${ }^{(1)}$.

O linfedema, principal complicação que ocorre nas mulheres com câncer de mama, pode ser decorrente de linfadenectomia e/ou ressecção de vasos linfáticos, metástase de tumores malignos, fibrose pósradioterapia e radiodermite, sendo prejudicial à função do membro homolateral, comprometendo a estética da paciente, podendo ter como consequência a dor ${ }^{(7)}$. 
Em períodos tardios do pós-operatório, acontece com maior frequência a dor residual, que, na maioria dos casos, tem origem em músculos, tendões, fáscias e pode apresentar a dor referida. A principal causa da dor no tratamento do câncer de mama relaciona-se à limitação de movimentos ocasionada por retrações músculo-aponeuróticas, consequentes à falta de exercícios físicos ${ }^{(7)}$.

Assim, o Projeto MUCAMA apoia-se nesta queixa mais recorrente e orienta quanto à realização de exercícios físicos individuais adequados à situação da mulher mastectomizada, além de oferecer atividade física em grupo, avalizada por fisioterapeutas, em um ambiente relaxante e com presença de música e assistência de enfermagem na prevenção da dor e de sequelas.

Neste contexto, o presente estudo teve como objetivo avaliar, de forma multidimensional e sistemática, as queixas álgicas relatadas pelas usuárias do Projeto MUCAMA, sendo utilizada a escala de MacGuill com a finalidade de detectar a dor nos aspectos avaliativo e de intensidade, e o período em que surge, de modo que a equipe promova atendimento que leve ao maior conforto às pacientes.

\section{METODOLOGIA}

O presente estudo é descritivo e de natureza quantitativa, do tipo exploratório e de delineamento transversal. Utilizou-se formulário para avaliar a dor quanto à descrição, à intensidade e à qualidade da queixa álgica - por meio da escala de McGuill - e fatores relacionados à mesma.

A escala foi aplicada a 20 mulheres com câncer de mama cadastradas no Núcleo de Ensino, Pesquisa e Extensão Mulher e Câncer de Mama da UNIFAL-MG, localizado no Município de Alfenas, Minas Gerais, no período de junho a setembro de 2008. Foram fatores de inclusão ter sido submetida à exérese de rede linfática axilar, ter concluído o tratamento adjuvante e concordar formalmente em participar da pesquisa.

O formulário utilizado na coleta de dados foi aplicado durante as reuniões do projeto, que ocorrem em um espaço próprio para esta atividade na Universidade, e complementado pela consulta ao prontuário. $\mathrm{O}$ instrumento de McGuill foi escolhido por apresentar uma dimensão ampla da queixa álgica, possibilitando às entrevistadas maior expressão de seus sentimentos.

Os dados foram coletados pelas autoras após a aprovação do projeto pelo Comitê de Ética em Pesquisa da Universidade Federal de Alfenas, sob o protocolo
23087.002621/2008-86. As participantes foram informadas acerca do objetivo da pesquisa e assinaram o Termo de Consentimento Livre e Esclarecido de forma voluntária, garantindo-lhes a identidade preservada, a desistência da pesquisa a qualquer momento e o acesso às informações sobre a publicação dos resultados.

As informações obtidas durante a entrevista foram organizadas em tabelas e gráficos e sofreram análise estatística simples para a discussão. Ressaltase que as mulheres podiam escolher nenhum, um ou mais itens em cada categoria que pontua aspectos sobre a qualidade da dor, os quais são: sensitiva, avaliativa e emocional.

\section{RESULTADOS E DISCUSSÃO}

O grupo foi formado por mulheres com idade acima de 40 anos, sendo que $70 \%$ tinham mais de 50 anos de idade, sendo importante registrar que o câncer de mama é uma patologia frequente após esta faixa etária. Apesar disso, encontramos um grupo com idade inferior aos 50 anos, o que significa que a prática de rastreamento do câncer, a partir dos 40 anos, com a finalidade de vigilância e para descoberta de casos em estágios iniciais e, portanto, com maior possibilidade de cura, tem sido cumprida pelo Município de Alfenas, que oferece atendimento pelo serviço público de saúde ${ }^{(1,10)}$.

Esse grupo de mulheres tem como ocupação trabalhos típicos do sexo feminino, $30 \%$ são aposentadas e $30 \%$ com baixa escolaridade. Esses fatores explicam a busca pelo serviço do Projeto universitário MUCAMA, tendo em vista a baixa renda, a necessidade de informações e o atendimento especializado e gratuito.

Constatou-se, por meio das informações do prontuário, que $10 \%$ das mulheres realizaram cirurgia radical do tipo Halsted, $30 \%$ apresentaram mastectomia radical modificada do tipo Pattey, $15 \%$ do tipo Madden, $20 \%$ mastectomia simples com esvaziamento ganglionar, além de $15 \%$ tumorectomia e $10 \%$ quadrantectomia como cirurgias reparadoras. Uma dessas pacientes realizou mastectomia bilateral e outra está em estágio de metástase comprovada por exames.

Ao relacionar o tipo de procedimento cirúrgico realizado com a descoberta do câncer de mama, pode-se notar que, quanto maior o tempo transcorrido do desenvolvimento e invasão da neoplasia até o diagnóstico, mais radical foi o procedimento. Esse fato coincide com outros estudos, pois, quanto mais 
precocemente se faz a descoberta, menor é o estadio e menos mutilante é a cirurgia ${ }^{(1,10)}$.

Observou-se que na população de nosso estudo, tal como na população brasileira em geral, a maioria dos diagnósticos de câncer de mama foi realizada a partir do estádio III $^{(1,11)}$.

O câncer de mama é uma patologia considerada indolor na fase pré-clínica, contudo, a dor é uma queixa recorrente no decorrer da evolução da doençca ${ }^{(4)}$. Em nosso estudo, 50\% das entrevistadas não referiram queixa dolorosa, seja no diagnóstico, tratamento ou após alta oncológica. As demais relataram dor; $40 \%$ referiram sentir pouca dor, e somente uma mulher relatou dor insuportável. A dor no câncer de mama pode ser decorrente de aspectos físicos e psicológicos e a participação em um grupo de apoio pode ser fator determinante para um relato minimizado da queixa dolorosa ${ }^{(8,12)}$.

Quando questionadas sobre o período em que a dor ocorreu, $80 \%$ relataram ser na fase cirúrgica, independente de ser no período pré, trans ou pós-operatório, seguida pelo período do diagnóstico médico. Elas relacionaram a algia a um ou mais períodos do câncer de mama, sendo observado que 50\% citaram dor em um momento, $30 \%$ a relacionaram a duas etapas e $20 \%$ afirmaram ter sofrido dor em todas as fases da doença, abarcando, portanto, o diagnóstico, o tratamento e a recuperação.

Verificou-se, portanto, que a maioria das pacientes que relataram algia afirma que sua queixa esteve relacionada à cirurgia, dado que corrobora estudo que identificou a dor como a principal complicação pós-cirúrgica da mastectomia ${ }^{(2)}$. Contudo, no mesmo estudo, a algia foi pesquisada como uma complicação pós-operatória, o que pode ter acentuado a sensação da dor, situação diferente da encontrada neste estudo, em que as mulheres se encontram no pós-operatório tardio.

Quanto aos aspectos que avaliaram a dor, a mensuração ocorreu por meio da intensidade; o caráter qualitativo da escala foi escolhido por meio das categorias sensitiva, avaliativa e emocional, sendo quantificadas as variáveis descritas na escala de McGuill, o que proporcionou uma avaliação multidimensional da queixa dolorosa.

Os descritores sensitivos mais citados foram ferroa e fisga, com significados de aguilhoada, pontada e dor aguda e rápida ${ }^{(13)}$, o que caracterizou a análise sensitiva da dor no câncer de mama neste grupo (Tabela 1). Cólica, esmaga e racha não foram relacionados à percepção dolorosa das pacientes, uma vez que não foram relatados.
Tabela 1 - Distribuição dos descritores sensitivos da queixa álgica. Alfenas, 2008

\begin{tabular}{lcc}
\hline Descritores sensitivos* & $\mathbf{n}$ & $\mathbf{\%}$ \\
\hline Ferroa & 6 & 16,22 \\
Fisga & 6 & 16,22 \\
Lateja & 5 & 13,51 \\
Agulhada & 5 & 13,51 \\
Facada & 3 & 8,11 \\
Estica & 3 & 8,11 \\
Pesa & 3 & 8,11 \\
Aperta & 2 & 5,4 \\
Torção & 2 & 5,4 \\
Rasga & 2 & 5,4 \\
Total & 37 & 100 \\
\hline
\end{tabular}

*Não houve mais de uma resposta por entrevistada

Com relação ao enfrentamento das pacientes diante da experiência dolorosa no câncer de mama, adormece foi o descritor avaliativo predominante seguido por irradia (Tabela 2). A sensação de adormecimento pode ser consequência de lesão do nervo intercostobraquial, o que compromete a sensibilidade e movimentação do membro homolateral. Além disso, a tendência de as mulheres movimentarem pouco o braço compromete a circulação do mesmo(1).

Tabela 2 - Distribuição dos itens avaliativos da dor. Alfenas, 2008

\begin{tabular}{lcc}
\hline Descritores avaliativos* & $\mathbf{n}$ & $\mathbf{\%}$ \\
\hline Adormece & 7 & 20 \\
Irradia & 5 & 14,29 \\
Coça & 4 & 11,43 \\
Choque & 4 & 11,43 \\
Queima & 4 & 11,43 \\
Esquenta & 3 & 8,57 \\
Arde & 3 & 8,57 \\
Formiga & 3 & 8,57 \\
Esfria & 2 & 5,71 \\
Total & 35 & 100 \\
\hline
\end{tabular}

*Não houve mais de uma resposta por entrevistada

Por meio da descrição do sintoma estudado, item presente no formulário aplicado, verificou-se os locais em que houve irradiação da dor. A maioria das mulheres submetidas a cirurgias da mama relatou irradiação para o membro homolateral à cirurgia (55\%). Esse fato era esperado, pois a retirada da mama e da musculatura leva à diminuição da sensibilidade e à sensação de dor irradiada.

No entanto, verificou-se que mulheres que 
receberam orientação sobre o autocuidado com o braço operado e que não restringiram os movimentos do braço relataram menor queixa de dor. Sabe-se que a Enfermagem tem um papel importante no pós operatório da cirurgia de mama, no sentido de orientação da mulher para que, no período de pós-alta hospitalar, a mesma tenha consciência de que é importante realizar pequenos movimentos até o altura da cabeça, para auxiliar o funcionamento do dreno à vácuo e impedir a limitação dos membros, entre outros fatores. Após a retirada do dreno e dos pontos, a mulher deve ser encaminhada a grupos de ajuda ou a fisioterapia.

Quanto à avaliação dos descritores emocionais (Tabela 3), o sentimento incômoda foi o prevalente, o que denota $\mathrm{o}$ aspecto psicológico do câncer. No entanto, os descritores agonizante e terror não foram citados. Essa postura demonstra que o sofrimento psicológico é representativo na vida da mulher com câncer de mama, mas, com apoio de um grupo especializado e dos familiares, essa queixa pode ser minimizada.

Tabela 3 - Distribuição dos descritores emocionais da queixa álgica. Alfenas, 2008

\begin{tabular}{lcc}
\hline Descritores emocionais* & n & $\mathbf{\%}$ \\
\hline Incômoda & 6 & 17,65 \\
Enjoada & 5 & 14,71 \\
Chata & 5 & 14,71 \\
Desgastante & 5 & 14,71 \\
Insuportável & 3 & 8,82 \\
Cansativa & 3 & 8,82 \\
Cruel & 2 & 5,88 \\
Torturante & 2 & 5,88 \\
Castigante & 1 & 2,94 \\
Aborrecida & 1 & 2,94 \\
Total & 33 & 100 \\
\hline
\end{tabular}

*Não houve mais de uma resposta por entrevistada

Em relação ao câncer, observa-se que, embora tenham aumentado as possibilidades terapêuticas, este ainda se apresenta no imaginário social como algo que cresce e destrói. É visto como uma doença cruel, intratável e misteriosa, que compromete um órgão representativo da função social do corpo da mulher, da identidade feminina, afetando papéis na sexualidade e sensualidade, esta dor pode se acentuar ${ }^{(14)}$. A dor emocional foi citada por todas as participantes.

O quadro 1 relaciona os fatores de enfrentamento, fracasso e de início da dor e a importância de fornecer ao profissional conhecimento acerca da dimensão da queixa dolorosa no grupo estudado.
Nos itens de melhora, prevalece a utilização de medidas farmacológicas; no entanto, a realização de exercícios fisioterapêuticos aparece em seguida, o que demonstra o reconhecimento da eficácia de métodos não medicamentosos. Nos fatores de piora, o excesso de exercícios físicos e a movimentação corporal relacionadas à tarefa doméstica são predominantes e apareceram com a mesma frequência, o que demonstra que, apesar de conhecer os aspectos negativos dessas atividades, as mulheres tendem a realizá-las, na maioria das vezes por não terem quem o faça. Quanto aos motivos que desencadeiam a dor, o item indeterminado apareceu em maior proporção e indica que o início da dor não está relacionado a um ato conhecido, mas com as atividades relacionadas ao excesso de trabalho e mudanças de posição, que estão associadas ao descritor de piora da dor.

Quadro 1 - Fatores relacionados à dor divididos quanto à melhora, à piora e ao motivo que desencadeia a algia*. Alfenas, 2008

\begin{tabular}{|lcc|}
\hline MELHORA & $\mathbf{n}$ & $\mathbf{\%}$ \\
Medicação analgésica & 5 & 35,71 \\
Exercícios fisioterapêuticos & 4 & 28,57 \\
Nada & 3 & 21,44 \\
Colocar mãos para baixo & 1 & 7,14 \\
Enfaixamento terapêutico & 1 & 7,14 \\
Total & 14 & 100 \\
PIORA & & \\
Excesso exercício físico & 4 & 36,36 \\
Movimentação corporal & 4 & 36,36 \\
Tempo frio & 1 & 9,09 \\
Ao término da fisioterapia & 1 & 9,09 \\
Mal-estar físico & 1 & 9,09 \\
Total & 11 & 100 \\
DESENCADEIA & & \\
Indeterminado & 3 & 30 \\
Após trabalhar em excesso & 2 & 20 \\
Ao mudar de posição & 2 & 20 \\
Após cirurgia & 1 & 10 \\
Metástase & 1 & 10 \\
Debridamento devido necrose após cirurgia & 1 & 10 \\
Total & 10 & 100 \\
\hline
\end{tabular}

*Não houve mais de uma resposta por entrevistada

\section{CONCLUSÃO}

O estudo permitiu concluir que metade das pacientes entrevistadas apresentou queixa dolorosa, em algum momento da doença ou do tratamento, e 
que a mesma esteve relacionada principalmente com intervenção cirúrgica e exérese da rede linfática.

$\mathrm{O}$ formulário aplicado permitiu avaliar a dor em seus aspectos quantitativos e qualitativos, o que possibilitou uma abordagem multidimensional, além de proporcionar maiores conhecimentos acerca da dimensão da queixa dolorosa ao analisar a descrição e fatores relacionados à dor. Verificou-se que as categorias sensitiva, avaliativa e emocional foram citadas de maneira regular. Mulheres sem apoio familiar demonstraram maior instabilidade emocional.

A equipe de enfermagem deve estar capacitada para avaliar com eficácia a dor, seja em qualquer patologia, nas consultas de enfermagem, principalmente em pacientes com doenças oncológicas, melhorando a qualidade de vida e enfatizando o cuidado de enfermagem.

Uma avaliação de qualidade utilizando instrumentos adequados permitirá intervenções efetivas que possibilitem o bem-estar físico e emocional das mulheres com câncer, o que diminuirá a dimensão dolorosa. A partir deste estudo, a mensuração da dor se tornou uma rotina no atendimento às mulheres que frequentam o MUCAMA, e o despertar para a dor emocional ficou conscientizado pelo grupo, pois mesmo sem a retirada da mama, a mulher pode apresentar a queixa da dor.

\section{REFERÊNCIAS}

1. Smeltzer SC, Bare BG. Tratado de enfermagem médico-cirúrgica. $10^{\mathrm{a}}$ ed. Rio de Janeiro: Guanabara Koogan; 2005. v1

2. Souza VP, Panobianco MS, Almeida AM, Prado MAS, Santos MSM. Fatores predisponentes ao linfedema de braço referidos por mulheres mastectomizadas. Rev Enferm. UERJ. 2007;15(1):87-93.

3. Freitas F, Passos EP, Rivoire W. Rotinas em ginecologia. $5^{\text {a }}$ ed. Porto Alegre: Artmed; 2008.

4. Mohallem AGC, Rodrigues AB. Enfermagem oncológica. Barueri: Manole; 2007.

5. Rigotti MA, Ferreira AM. Intervenções de enfermagem ao paciente com dor. Arq Ciênc Saúde. 2005;12(1):50-4.

6. Pimenta CAM, Koizumi MS, Teixeira MJ. Dor no doente com câncer: características e controle. Rev Bras Cancerol. 1997;43(1):52-60.

7. Camargo MC, Marx AG. Reabilitação física no câncer de mama. São Paulo: Rocca; 2000.
8. Monteiro CFS, Veloso LUP, Sousa PCB, Morais SCRV. A vivência familiar diante do adoecimento e tratamento de crianças e adolescentes com leucemia linfóide aguda. Cogitare Enferm. 2008;13(4): 484-9.

9. Torresan RZ, Santos CC, Conde DM, Brenelli HB. Preservação do nervo intercostobraquial na linfadenectomia axilar por carcinoma de mama. Rev Bras Ginecol Obstetric. 2002;24(4):221-6.

10. Ministério da Saúde (BR). Instituto Nacional do Câncer. Controle do câncer de mama: documento do consenso. Rio de Janeiro: INCA; 2004.

11. Batiston AP, Tamaki EM, Santos MLM, Cazola LHO. Método de detecção do câncer de mama e suas implicações. Cogitare Enferm. 2009;14(1):59-64.

12. Siqueira KM, Barbosa MA, Boemer MR. O vivenciar a situação com câncer: alguns desvelamentos. Rev Latino-Am Enfermagem. 2007;15(4):605-11.

13. Ferreira $\mathrm{ABH}$ Novo dicionário Aurélio da língua portuguesa. 3 ed. Curitiba: Positivo; 2004.

14. Vieira RJS, Gomes R, Trajano AJB. Câncer de mama e gravidez subseqüente: um olhar sócio-cultural. Rev Bras Cancerol. 2005;51(2):101-10. 